\title{
Modelos teóricos y estudiantes de enfermería: aspectos motivacionales para su elección durante las prácticas clínicas
}

Susana Santos-Ruiz, María López-Parra, Guadalupe Sánchez-Rueda, Lola Bardallo-Porras, Lidia Fernández-Donaire, Adela Zabalegui-Yárnoz, Dolors Abril-Sabater, Susana Varez-Pelaez, Joaquín Tomás-Sábado

Introducción. El cuerpo teórico y filosófico de enfermería es complejo; existen un gran número de teorías enfermeras, algunas de difícil comprensión, por lo que habitualmente su aplicación práctica se ve restringida.

objetivo. Identificar el modelo teórico de enfermería que los estudiantes dicen utilizar durante las prácticas clínicas, la motivación para su elección, y la escuela universitaria donde estaban cursando sus estudios.

Sujetos y métodos. Estudio descriptivo en el que participaron 105 estudiantes (de una población total de 108) que realizaban prácticas de enfermería en el hospital durante el curso 2006-2007. La variables de estudio fueron los modelos teóricos enfermeros, las razones de la elección y variables sociodemográficas. La recogida de datos se llevo a cabo mediante un cuestionario cumplimentado durante su experiencia clínica. El análisis de los datos se realizó mediante un análisis temático de texto, categorizando para crear finalmente un marco explicativo.

Resultados y conclusiones. El modelo elegido es principalmente el de Virginia Henderson. Las motivaciones para la elección, según los estudiantes, son su uso en la enseñanza universitaria y la facilidad de ejecución del modelo. Se evidenció confusión entre los términos 'modelo conceptual y/o teórico' y 'metodología enfermera'.

Palabras clave. Estudiantes de enfermería. Modelos teóricos. Teoría de enfermería.

Theoretical models and nursing students: motivational aspects for election in clinical practice

Introduction. The theoretical and philosophical nursing is complex, there are a large number of nursing theories, some difficult to understand, because this usually its practical application is restricted.

Aim. To identify the theoretical model of nursing students say they use in clinical practice, the motivation for their choice, and school where they were pursuing their studies.

Subjects and methods. Descriptive study. Participants were 105 students doing school practice during the academic year 2006-2007 (of a total population of 108 students). The study variables were the nursing models and theories, the reasons for the choice and demographic variables. Data collection was conducted through a questionnaire completed during their clinical experience. The analysis of data using a thematic analysis of text, categorizing to finally create an explanatory framework.

Results and conclusions. The model chosen is mainly that of Virginia Henderson. The motivations for the election as students are used in university education and ease of implementation of the model. It was evident confusion between the terms related to nursing model and methodology.

Key words. Nursing students. Nursing theory. Theoretical models.

\section{Introducción}

Los modelos conceptuales se usan en múltiples disciplinas para poder explicar los fenómenos, definir fronteras profesionales y reflejar las creencias, valores y aspiraciones de las profesiones [1].
El cuerpo filosófico de enfermería es complejo, ya que existen un gran número de modelos conceptuales y teorías enfermeras, algunas de difícil comprensión [2]. Los modelos habitualmente tienden a centrarse en 'lo que debería ser', lo cual se opone a la visión de la realidad enfermera, en la que las profesio-
Escola Creu Roja; Universitat Autònoma de Barcelona, UAB; Terrassa, Barcelona (G. SánchezRueda). Universitat Internacional de Catalunya; Sant Cugat, Barcelona (L. Bardallo Porras). Hospital Clínic; Barcelona (A. ZabaleguiYárnoz). Escuela Universitaria de Enfermería Gimbernat; UAB; Sant Cugat, Barcelona (L. FernándezDonaire, J. Tomás-Sábado). Corporació Sanitària Parc Taulí; Sabadell, Barcelona (S. Santos-Ruiz, M. López-Parra, D. Abril-Sabater, S. Varez-Pelaez).

Correspondencia:

MsU. Susana Santos Ruiz. Corporació Sanitària Parc Taulí. Parc Taulí, s/n. E-08208 Sabadell (Barcelona).

E-mail:

susanasantosr@gmail.com

Agradecimientos:

A los estudiantes que respondieron al cuestionario y a los tutores de prácticas que facilitaron la distribución de los cuestionarios.

(c) 2011 Educación Médica 
Tabla I. Datos característicos de cada uno de los centros universitarios.

\begin{tabular}{|c|c|c|c|}
\hline & Escuela $1[18,19]$ & Escuela $2[20,21]$ & Universidad 3 [22-25] \\
\hline Tipo de institución & $\begin{array}{l}\text { Adscrita a la Universitat } \\
\text { Autònoma de Barcelona }\end{array}$ & $\begin{array}{l}\text { Adscrita a la Universitat } \\
\text { Autónoma de Barcelona }\end{array}$ & Entidad privada \\
\hline Horario & Mañana y tarde & Mañana & Mañana \\
\hline Alumnos totales en formación & 225 alumnos & 88 alumnos & 80 alumnos \\
\hline Filosofía del centro & $\begin{array}{l}\text { Perfil profesional enfermero que tenga como eje } \\
\text { el compromiso personal y profesional, y como } \\
\text { elementos clave, la capacidad de reflexionar y } \\
\text { de construir la propia práctica profesional en un } \\
\text { marco de necesidades y demandas cambiantes } \\
\text { donde persona y comunidad han de adquirir } \\
\text { protagonismo }\end{array}$ & $\begin{array}{l}\text { La actuación profesional transmitida se orienta } \\
\text { hacia la aplicación del Proceso enfermero y se } \\
\text { fomenta la utilización de técnicas e innovaciones } \\
\text { pedagógicas basadas en la interacción y la } \\
\text { participación que promueven la formación } \\
\text { integral y una rápida incorporación dentro del } \\
\text { mundo laboral }\end{array}$ & $\begin{array}{l}\text { El currículo enfatiza la investigación y el } \\
\text { liderazgo profesional (docencia, investigación } \\
\text { y aprendizaje) y se asienta en la interrelación } \\
\text { entre los conceptos 'persona', 'salud', } \\
\text { 'enfermería' y 'medioambiente', que son } \\
\text { a su vez la base de diferentes modelos de } \\
\text { enfermería }\end{array}$ \\
\hline $\begin{array}{l}\text { Modelo enfermero de } \\
\text { referencia para guiar } \\
\text { la docencia }\end{array}$ & Virginia Henderson & $\begin{array}{l}\text { No tienen modelo teórico referente; siguen } \\
\text { los patrones funcionales de salud de Marjory } \\
\text { Gordon }\end{array}$ & Sor Callista Roy \\
\hline $\begin{array}{l}\text { Asignatura donde se dan } \\
\text { contenidos filosóficos }\end{array}$ & $\begin{array}{l}\text { Fundamentos de Enfermería } \\
\text { (asignatura troncal, anual) }\end{array}$ & $\begin{array}{l}\text { Fundamentos de Enfermería } \\
\text { (asignatura troncal) }\end{array}$ & Fundamentos de Enfermería \\
\hline Créditos & 15 créditos (8 teóricos y 7 prácticos) & 15 créditos (teórico-prácticos) & 15 créditos teórico-prácticos \\
\hline Contenido de la asignatura & $\begin{array}{l}\text { Metaparadigma y escuelas de pensamiento } \\
\text { enfermero, modelo de V. Henderson, método } \\
\text { de trabajo enfermero, procedimientos } \\
\text { enfermeros y estancias prácticas }\end{array}$ & $\begin{array}{l}\text { Modelo holístico de salud, inspirado en los } 11 \\
\text { patrones funcionales de salud de M. Gordon } \\
\text { y su aplicación en el proceso enfermero. } \\
\text { Aportaciones teóricas y filosóficas de } \\
\text { la disciplina }\end{array}$ & $\begin{array}{l}\text { Aunque Roy es el marco referencial, } \\
\text { en el primer trimestre de la asignatura se } \\
\text { exponen también las bases de otras teóricas. } \\
\text { Desarrollo del proceso de atención de } \\
\text { enfermería }\end{array}$ \\
\hline
\end{tabular}

nales de base se preocupan por su labor diaria, adaptando los recursos disponibles a sus necesidades. En general se observa que las enfermeras consideran habitualmente utópica su aplicación [3] y cuando realmente se implementan se realiza a través de un modelo enfermero sustitutivo o funcional [4].

La incorporación de los estudios de enfermería a la universidad se produjo a finales de los años setenta. Anteriormente existía la titulación de 'Ayudante Técnico Sanitario' (ATS), que como su nombre indica, reflejaba la supeditación al cuerpo médico con un rol totalmente colaborador, sin un cuerpo teórico propio [5]. Muchos de los profesionales todavía en activo en la actualidad fueron formados bajo el plan de estudios anterior y a pesar de que la mayoría convalidaron su titulación, su base teórica y filosófica sobre la profesión es muy distinta a la que se está impartiendo actualmente en las aulas [6,7].

Los modelos y teorías de enfermería se han trabajado históricamente en la universidad con diferente intensidad [8] y enfoque y quizá por este motivo existe poco acuerdo entre los profesionales de enfermería en relación a la necesidad de basar la práctica cuidadora en un modelo teórico explícito y claramente integrado. Los modelos enfermeros, actualmente, se imparten en el primer año de estudios de enfermería, con una dedicación de créditos ECTS (European Credits Transfer System) que oscila entre 5 y 20 [9].

El modelo de Virginia Henderson ha sido el más usado en la formación universitaria española durante la década de los ochenta y noventa [10]. Actualmente, a partir del año 2000, existe una mayor pluralidad formativa al respecto, pero el conocimiento que se transmite, según indica Medina [11], es tachado de abstracto, útil sólo en algunos casos concretos y la realidad práctica es inestable, incierta, compleja y saturada de opciones de valor.

La metodología educativa evoluciona y el profesorado realiza un esfuerzo considerable para desarrollar una docencia reflexiva y crítica, pero la realidad evidencia que, mientras no exista una transferencia del conocimiento teórico de las aulas a la práctica asistencial, será difícil conseguir la integra- 
ción de los modelos enfermeros de forma explícita en el cuidado de las personas [8].

A esto se añade la posibilidad de influencia de múltiples modelos formales en cada profesional [12]; no olvidemos que un solo modelo puede no dar respuesta a la alta complejidad de las respuestas humanas a la salud $[13,14]$, por lo que deberemos explotar las múltiples perspectivas [12].

Según Maben et al [7], los estudiantes acaban su formación universitaria con fuertes valores y conocimientos enfermeros que reflejan las teorías académicas promovidas por la educación enfermera, pero gran número de factores impiden ponerlos en práctica de forma completa y clara a causa del dominio del modelo biomédico, la estructura organizativa de las instituciones [6,7], la imposición de modelos [3], la elevada presión asistencial en las unidades de trabajo [7,15], la propia complejidad de los modelos $[2,11]$ y la existencia de 'reglas encubiertas' por las que son socializados los profesionales según las necesidades de las instituciones, de forma que prevalece el cuidado de aspectos físicos sobre el resto [7]. Las enfermeras, en ocasiones, se preocupan poco por la aplicación de metodología enfermera (relacionada directamente con el uso de modelos) en el cuidado, dando mayor relevancia a las habilidades técnicas y al dominio de procedimientos sin echar de menos el desarrollo del rol autónomo [16].

Para el establecimiento de un modelo en la práctica resulta básico que exista un proceso de reflexión y un importante trabajo de consenso [17]. Las investigaciones basadas en la opinión de las enfermeras asistenciales hospitalarias en lo referente a filosofía son escasas, hecho sorprendente porque, como dice Mckenna [3], se consideran internacionalmente la base para cuidar, y ponen de manifiesto la importancia de la participación activa en el tema de los propios profesionales [7].

Debido a que la corporación sanitaria objeto de estudio carecía de un modelo de enfermería para la práctica enfermera, surgió el interés de conocer la perspectiva que los estudiantes, futuros profesionales, mostraban sobre el tema. La bibliografía revisada no muestra estudios que analicen la opinión de los estudiantes en relación a la elección de un modelo de enfermería.

El objetivo del presente trabajo es:

- Identificar el modelo teórico de enfermería que los estudiantes dicen utilizar para la práctica asistencial.

- Describir los motivos de los estudiantes de dicha elección.

- Establecer la relación del modelo elegido con la escuela en la que estudian los alumnos.
Figura 1. Cuestionario para estudiantes de enfermería.

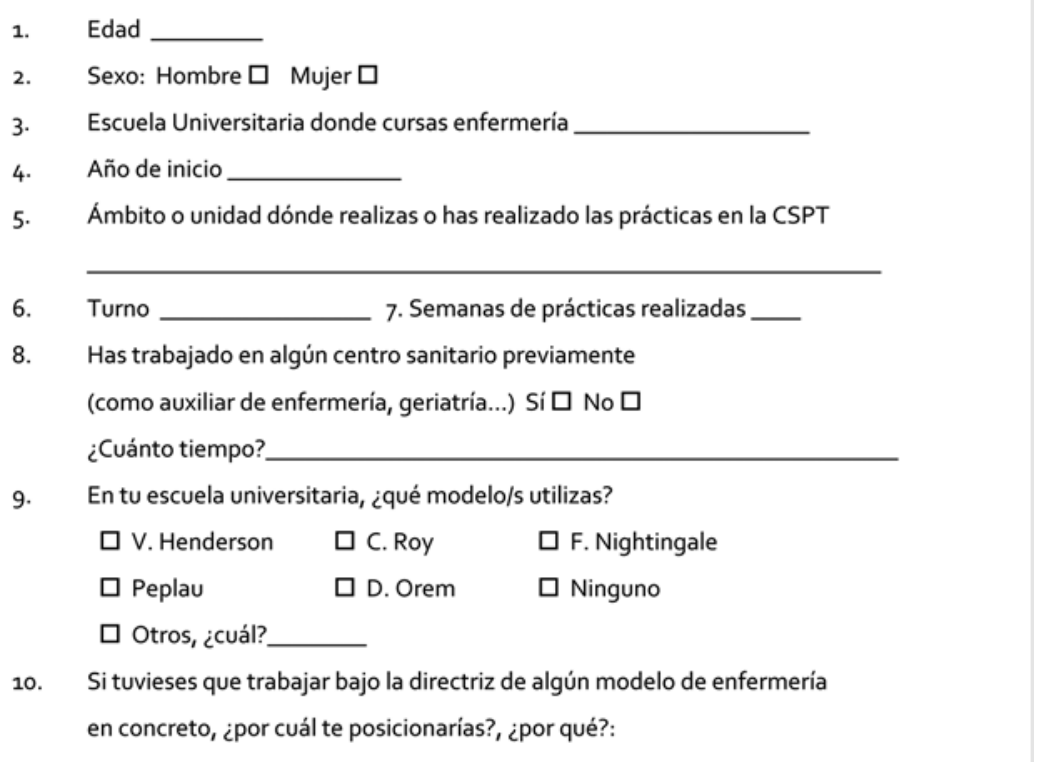

\section{Sujetos y métodos}

Se trata de un estudio descriptivo, transversal. La población de estudio fueron la totalidad de los estudiantes de las tres escuelas universitarias participantes que realizaban sus prácticas en la Corporació Sanitària Parc Taulí (Sabadell, Barcelona) durante el curso 2006-2007. Fueron un total de 105 estudiantes (de los 108 potenciales) quienes dieron su consentimiento para participar voluntariamente en el estudio. Los criterios de inclusión fueron alumnos de los tres cursos de la diplomatura que estuviesen realizando las prácticas en la corporación.

Las tres escuelas que aportan los estudiantes de prácticas tienen ciertas características que las diferencian en cuanto a la asignatura de 'Fundamentos de Enfermería' y el modelo enfermero en el cual basan su formación. Para facilitar la lectura del texto, se numerarán cada una de las escuelas:

- Número 1: Escuela de Enfermería Creu Roja (Terrassa).

- Número 2: Escuela de Enfermería Gimbernat (Sant Cugat).

- Número 3: Facultad de Ciencias de la Salud, perteneciente a la Universitat Internacional de Catalunya (Sant Cugat). 
Tabla II. Datos demográficos de la muestra.

\begin{tabular}{|c|c|c|c|c|}
\hline & Opciones de respuesta & Escuela $1(n=45)$ & Escuela $2(n=30)$ & Universidad $3(n=30)$ \\
\hline Edad del estudiante & Media (años) & $24,16 \pm 6,35$ & $22,83 \pm 4,14$ & $25,53 \pm 7,20$ \\
\hline Sexo del estudiante & Varones & $13,3 \%(n=6)$ & $13,3 \%(n=4)$ & $23,3 \%(n=7)$ \\
\hline Año de inicio de la carrera & & $2002-2006$ & $2003-2006$ & $2004-2006$ \\
\hline \multirow{7}{*}{$\begin{array}{l}\text { Ámbito o unidad } \\
\text { de prácticas }\end{array}$} & Cirugía & $31,1 \%(n=14)$ & $53,3 \%(n=16)$ & $13,3 \%(n=4)$ \\
\hline & Críticos & $8,9 \%(n=4)$ & $3,3 \%(n=1)$ & $30 \%(n=9)$ \\
\hline & Maternoinfantil & $28,9 \%(n=13)$ & $20 \%(n=6)$ & $10 \%(n=3)$ \\
\hline & Sociosanitario & $13,3 \%(n=6)$ & $10 \%(n=3)$ & $36,7 \%(n=11)$ \\
\hline & Medicina Interna & $11,1 \%(n=5)$ & $6,7 \%(n=2)$ & $6,7 \%(n=2)$ \\
\hline & Nefrología & 0 & 0 & $3,3 \%(n=1)$ \\
\hline & En blanco & $6,7 \%(n=3)$ & 0 & 0 \\
\hline Turno de prácticas & Mañana & $68,2 \%(n=30)$ & $100 \%(n=30)$ & $13,3 \%(n=4)$ \\
\hline $\begin{array}{l}\text { Semanas de prácticas } \\
\text { realizadas }\end{array}$ & 4 semanas & $51,1 \%(n=23)$ & $76,7 \%(n=23)$ & $83,3 \%(n=25)$ \\
\hline $\begin{array}{l}\text { Ha trabajado en algún centro } \\
\text { sanitario previamente }\end{array}$ & Sí & $\begin{array}{c}44,4 \%(n=20) \\
(60 \% \text { más de } 2 \text { años })\end{array}$ & $\begin{array}{c}60 \%(n=18) \\
\text { (57\% más de } 2 \text { años) }\end{array}$ & $\begin{array}{c}40 \%(n=12) \\
(18,2 \% \text { más de } 2 \text { años }\end{array}$ \\
\hline \multirow{6}{*}{$\begin{array}{l}\text { Modelo enfermero utilizado } \\
\text { en su escuela }\end{array}$} & Henderson & $100 \%(n=45)$ & $46,7 \%(n=14)$ & $13,8 \%(n=4)$ \\
\hline & Roy & 0 & 0 & $86,2 \%(n=25)$ \\
\hline & Nightingale & 0 & $3,3 \%(n=1)$ & 0 \\
\hline & Ninguno & 0 & $3,3 \%(n=1)$ & 0 \\
\hline & Otros & 0 & $46,7 \%(n=14)$ & 0 \\
\hline & No contesta & 0 & 0 & $3,3 \%(n=1)$ \\
\hline
\end{tabular}

Las principales características de cada uno de los centros se resumen en la tabla I.

La recogida de datos se realizó en la Corporació Sanitària Parc Taulí. En el momento de la recogida de los datos, el centro se hallaba en un momento de transición tanto en el aspecto informático como en el de la definición y desarrollo de una nueva metodología de trabajo a través de la incorporación de metodología enfermera basada en planes de cuidados.

\section{Variables de estudio}

- Variable principal: modelos y teorías enfermeras, y motivos de elección. Se entiende por modelos y teorías de enfermería el uso de las bases filosóficas enfermeras en el proceso de cuidar. El motivo de elección será aquella característica del modelo que decanta su designación.

- Variables secundarias: edad, experiencia laboral previa, escuela y modelo enfermero utilizado en la escuela de enfermería.

\section{Recogida de datos}

Se utilizó un cuestionario anónimo elaborado por los autores, que fue entregado en mano a los estu- 
diantes por el equipo investigador en una sesión de tutoría durante el periodo de prácticas. La recogida se realizó en la siguiente sesión de tutoría por parte de la monitora de prácticas. El cuestionario fue autoadministrado, fuera de horario de prácticas, sin presencia de tutores o equipo investigador. Se realizó una prueba piloto con 17 estudiantes para comprobar su comprensión. No se creyó necesaria la validación del cuestionario porque el objetivo era obtener una información de carácter cualitativo.

El cuestionario recogía las variables de estudio y una pregunta final abierta que recogía la variable principal (Figura 1).

\section{Análisis de los datos}

Se realizó el recuento de los modelos propuestos, expresándose los resultados en forma de porcentajes. Para la segunda parte de la pregunta se realizó un análisis de tipo cualitativo: análisis temático de las respuestas escritas por los estudiantes (trascripción literal, lectura repetida y clasificación provisional de temas, codificación, categorías, verificación y contraste) para la creación de un marco explicativo.

\section{Resultados}

Participaron 105 estudiantes: 45 alumnos de la escuela 1, 30 alumnos de la escuela 2 y 30 de la universidad 3. El índice de participación fue del 98\%. Los datos demográficos se presentan en la tabla II.

En cuanto al modelo enfermero propuesto para su práctica (Tabla III), el modelo más escogido fue el de V. Henderson (50,5\%); no obstante, el 26,7\% dejaron la pregunta en blanco y un $1,9 \%$ indicaron que no querían trabajar bajo la directriz de un modelo enfermero. Era de destacar que el 13,3\% indicaban metodologías de trabajo que no eran modelos teóricos enfermeros (M. Gordon, NANDA, PAE).

Cuando analizamos los datos según la escuela, el modelo de elección es el de V. Henderson para el $73,3 \%$ de los estudiantes de la escuela 1, el 30\% de los de la escuela 2 (aunque son los patrones funcionales de Marjory Gordon para el 26,6\%) y el 36,6\% de los alumnos de la universidad 3 (no obstante, el 46,6\% dejaron la pregunta en blanco).

Los estudiantes dicen elegir un modelo teórico enfermero principalmente por ser el modelo usado en su formación universitaria y por la facilidad para su aplicación. En concreto, mayoritariamente optan por el de V. Henderson porque engloba a la persona
Tabla III. Modelo elegido para la práctica diaria.

\begin{tabular}{lcccc}
\hline & $\begin{array}{c}\text { Escuela 1 } \\
(n=45)\end{array}$ & $\begin{array}{c}\text { Escuela 2 } \\
(n=30)\end{array}$ & $\begin{array}{c}\text { Universidad 3 } \\
(n=30)\end{array}$ & $\begin{array}{c}\text { Total } \\
(n=105)\end{array}$ \\
\hline V. Henderson & 33 & 9 & 11 & $53(50,5 \%)$ \\
\hline C. Roy & 0 & 0 & 3 & $3(2,9 \%)$ \\
\hline V. Henderson + C. Roy & 0 & 0 & 2 & $2(1,9 \%)$ \\
\hline H. Peplau & 0 & 2 & 0 & $2(1,9 \%)$ \\
\hline En blanco & 9 & 5 & 14 & $28(26,7 \%)$ \\
\hline M. Gordon & 0 & 8 & 0 & $8(7,6 \%)$ \\
\hline 'Humanista' & 3 & 0 & 0 & $3(2,9 \%)$ \\
\hline NANDA & 0 & 2 & 0 & $2(1,9 \%)$ \\
\hline Ninguno & 0 & 2 & 0 & $2(1,9 \%)$ \\
\hline PAE & 0 & 2 & 0 & 2 \\
\hline
\end{tabular}

NANDA: North American Nursing Diagnosis Association; PAE: proceso de atención de enfermería.

con sus catorce necesidades, porque resulta importante la valoración de la persona y porque es holístico. No obstante, indican también que les gustaría tener mayor formación sobre el tema.

Quienes dejan la pregunta en blanco (se desconoce si es sinónimo de indicar 'ninguno'), en su mayoría no indican el porqué de su abstención. En un pequeño número indican que no optan por un modelo ya que optarían por uno en concreto según las características de la persona a cuidar (estudiantes de la escuela 2 y de la universidad 3) o prefieren la combinación de varios para la práctica (estudiantes de la escuela 2).

Existe un numero importante de estudiantes que optan por metodologías de trabajo y no por modelos enfermeros, aunque no indican que la opción elegida no sea un modelo enfermero. Entre opciones que no son modelos se escogen los patrones de M. Gordon, porque es la opción que recibieron en su formación; el PAE, porque 'es estructurado' y 'engloba las catorce necesidades', y el NANDA, por haberlo usado en su formación (estudiantes de la escuela 2).

Los textos según las categorías halladas del análisis cualitativo se resumen en las tablas IV y V. Tras la lectura repetida del texto escrito por los alumnos se llegó a la conclusión de que se podían agrupar los comentarios en las siguientes categorías: expresiones relacionadas con el modelo, características 
Tabla IV. Comentarios recogidos en los cuestionarios a la pregunta de por qué habían indicado el modelo de V. Henderson (se muestran los escritos textuales de los estudiantes recogidos del texto que son demostrativos de las categorías halladas).

\begin{tabular}{|c|c|c|c|c|c|}
\hline & \multicolumn{5}{|c|}{ Categorías halladas } \\
\hline & $\begin{array}{l}\text { Expresiones verbales } \\
\text { relacionadas con el modelo }\end{array}$ & $\begin{array}{l}\text { Características referidas } \\
\text { del modelo elegido }\end{array}$ & $\begin{array}{l}\text { Expresiones verbales } \\
\text { relacionadas con el } \\
\text { encuestado }\end{array}$ & $\begin{array}{l}\text { Expresiones verbales } \\
\text { relacionadas con la } \\
\text { escuela }\end{array}$ & Otros \\
\hline Escuela 1 & $\begin{array}{l}\text { Engloba } \\
\text { Se ve claro } \\
\text { Permite valorar } \\
\text { Aborda, explora, abarca, } \\
\text { valora necesidades } \\
\text { No despersonaliza } \\
\text { No deshumaniza }\end{array}$ & $\begin{array}{l}\text { Holístico } \\
\text { Claridad } \\
\text { Fácil } \\
\text { Útil } \\
\text { Completo } \\
\text { Funciona } \\
\text { Biopsicosocial } \\
\text { Humanista } \\
\text { Único estudiado }\end{array}$ & $\begin{array}{l}\text { Conozco } \\
\text { No conozco otros } \\
\text { He estudiado } \\
\text { He utilizado } \\
\text { He practicado } \\
\text { He trabajado } \\
\text { He aplicado } \\
\text { He visto utilizar }\end{array}$ & $\begin{array}{l}\text { Se inculca } \\
\text { Se imparte } \\
\text { Se enseña } \\
\text { Se profundiza } \\
\text { Se basa la carrera }\end{array}$ & $\begin{array}{l}\text { Me gustaría } \\
\text { conocer otros } \\
\text { Me gustaría poder } \\
\text { trabajar con otros } \\
\text { No tengo } \\
\text { conocimiento } \\
\text { para valorar otros }\end{array}$ \\
\hline Escuela 2 & $\begin{array}{l}\text { Tiene en cuenta } \\
\text { Contempla } \\
\text { Recoge datos } \\
\text { Facilita el diagnóstico } \\
\text { Favorece la labor enfermera } \\
\text { Engloba } \\
\text { Se aproxima a necesidades } \\
\text { Se acerca } \\
\text { No olvida aspectos vitales }\end{array}$ & $\begin{array}{l}\text { Más utilizado, más conocido } \\
\text { Observaciones básicas } \\
\text { Buen modelo } \\
\text { Completo, estructurado, ordenado, } \\
\text { planificado, divido por partes, lineal } \\
\text { Fácil } \\
\text { Holístico }\end{array}$ & $\begin{array}{l}\text { Conozco } \\
\text { He utilizado } \\
\text { Hemos trabajado }\end{array}$ & $\begin{array}{l}\text { Es el referente } \\
\text { de la escuela }\end{array}$ & $\begin{array}{l}\text { Redactaría de } \\
\text { otra forma } \\
\text { las necesidades } \\
\text { Considera } \\
\text { los patrones }\end{array}$ \\
\hline Universidad 3 & $\begin{array}{l}\text { Embarca todas la dimensiones } \\
\text { Cubre } 14 \text { necesidades, las necesidades básicas } \\
\text { Incluye, abarca, tiene en cuenta todos } \\
\text { los aspectos } \\
\text { Sigue un orden } \\
\text { Evita valoración regular e incompleta, } \\
\text { descuidar necesidades, es una forma } \\
\text { de valorar y registrar } \\
\text { Enfocado para todo tipo de persona }\end{array}$ & $\begin{array}{l}\text { Rápido } \\
\text { Fácil de valorar } \\
\text { Amplio } \\
\text { Sencillo para la práctica } \\
\text { Práctico } \\
\text { Completo } \\
\text { Fácil } \\
\text { Claro } \\
\text { Conciso } \\
\text { Completo para valoración y seguimiento }\end{array}$ & $\begin{array}{l}\text { Considero de } \\
\text { sencilla aplicación }\end{array}$ & & $\begin{array}{l}\text { La falta de tiempo } \\
\text { no permite usar } \\
\text { otros modelos }\end{array}$ \\
\hline
\end{tabular}

del modelo, expresiones relacionadas con el alumno y expresiones relacionadas con la escuela. Hubo información que no pudo incluirse en dichas categorías. Esta miscelánea se agrupa dentro de 'otros'. Este análisis se fragmentó según la elección de las autoras y las escuelas. Los datos en referencia al modelo elegido y su casuística se resumen en el mapa conceptual de la figura 2 .

\section{Discusión}

Globalmente podríamos sintetizar que la motivación para elegir un modelo o metodología se resumió en tres causas: orientación (conocimiento), dominio (facilidad de aplicación) y adecuación (características del propio modelo o metodología).

Pese a que se nombraron varias teóricas, fue Henderson la escogida por más de la mitad de los en- cuestados, aunque en dos de las facultades (escuela 2 y universidad 3) no es la teórica de referencia. Seguramente esta decisión estaba condicionada por los medios científicos de comunicación, en los que predomina el modelo de esta autora en las publicaciones relacionadas con planes de cuidados, sobre todo asociada a taxonomía NANDA, NIC y NOC [26-28]. Se observó una comprensión deficiente de la filosofía del modelo y un conocimiento parcial de éste; se repiten frases como 'es el que más he usado,' 'conozco' y ' 14 necesidades', pero escasamente comentan sobre manifestaciones de dependencia/independencia de la persona y no ilustran el papel que desempeña la enfermera dentro del modelo. En este caso podrían haber argumentado, por ejemplo, la elección de Henderson en base a cómo la enfermera realiza las acciones que el paciente no puede efectuar en un determinado momento de su ciclo de vital, fomentando, en mayor o menor grado, la independencia 
Tabla V. Comentarios recogidos en los cuestionarios a la pregunta de por qué habían indicado otros modelos teóricos o metodologías de trabajo diferentes al modelo de V. Henderson (se muestran los escritos textuales de los estudiantes recogidos del texto que son demostrativos de las categorías halladas).

\begin{tabular}{|c|c|c|c|c|c|c|}
\hline & Respuesta a & $\begin{array}{l}\text { Expresiones verbales } \\
\text { relacionadas con el modelo }\end{array}$ & $\begin{array}{l}\text { Características referidas } \\
\text { del modelo elegido }\end{array}$ & $\begin{array}{l}\text { Expresiones verbales } \\
\text { relacionadas con el } \\
\text { encuestado }\end{array}$ & $\begin{array}{l}\text { Expresiones verbales } \\
\text { relacionadas con la } \\
\text { escuela }\end{array}$ & Otros \\
\hline Escuela 1 & Modelo humanista & $\begin{array}{l}\text { No deshumaniza } \\
\text { No despersonaliza } \\
\text { Hacer mejor seguimiento } \\
\text { a cada persona }\end{array}$ & & & & Enfermería de roles \\
\hline \multirow{4}{*}{ Escuela 2} & $\begin{array}{l}\text { H. Peplau } \\
\text { (no se elige en } \\
\text { ninguna otra escuela) }\end{array}$ & $\begin{array}{l}\text { Orienta } \\
\text { Identifica problemas de salud }\end{array}$ & $\begin{array}{l}\text { Adecuado } \\
\text { Relación terapéutica } \\
\text { fundamental }\end{array}$ & Me parece más adecuado & & \\
\hline & M. Gordon & $\begin{array}{l}\text { Recoge toda la información } \\
\text { Tiene en cuenta la } \\
\text { mayoría de problemas } \\
\text { Engloba todos los aspectos } \\
\text { que puedan afectar } \\
\text { el bienestar } \\
\text { Engloba }\end{array}$ & $\begin{array}{l}\text { Mejor cuidado } \\
\text { Holístico } \\
\text { Sencillo } \\
\text { Virtudes y defectos }\end{array}$ & $\begin{array}{l}\text { He estudiado } \\
\text { Conozco } \\
\text { Hemos utilizado } \\
\text { Estoy habituado a } \\
\text { Conozco a fondo } \\
\text { Capaz de aplicar }\end{array}$ & $\begin{array}{l}\text { Nos enseñan } \\
\text { Trabajado en } \\
\text { la universidad } \\
\text { Utilizado en } \\
\text { la carrera }\end{array}$ & $\begin{array}{l}\text { Lo mejor sería hacer } \\
\text { un modelo con lo } \\
\text { mejor de todos } \\
\text { Cada modelo es útil } \\
\text { No tendría problema } \\
\text { en trabajar con otros }\end{array}$ \\
\hline & NANDA & & & Lo llevo haciendo tres años & & \\
\hline & PAE & $\begin{array}{l}\text { Tiene etapas } \\
\text { Recoge datos esenciales }\end{array}$ & $\begin{array}{l}\text { Estructurado } \\
\text { Definido }\end{array}$ & Tenemos una visión holística & & \\
\hline \multirow{2}{*}{ Universidad 3} & $\begin{array}{l}\text { C. Roy } \\
\text { (no se elige en } \\
\text { ninguna otra escuela) }\end{array}$ & $\begin{array}{l}\text { Complementa } \\
\text { Evita descuidar } \\
\text { Cubre necesidades, entorno } \\
\text { y condicionantes }\end{array}$ & $\begin{array}{l}\text { Empatía por el paciente } \\
\text { Completo }\end{array}$ & $\begin{array}{l}\text { Cogería un poco de Roy } \\
\text { y un poco de Henderson }\end{array}$ & & $\begin{array}{l}\text { Combinar } \\
\text { con Henderson } \\
\text { Con Henderson son } \\
\text { prácticos y completos }\end{array}$ \\
\hline & C. Roy + V. Henderson & & & & & $\begin{array}{l}\text { Complementa } \\
\text { y especifica } \\
\text { Prácticos y necesarios } \\
\text { para no descuidar }\end{array}$ \\
\hline
\end{tabular}

NANDA: North American Nursing Diagnosis Association; PAE: proceso de atención de enfermería.

del paciente [29]. Pese a ello, en algunos estudios en los que los profesionales escogieron un modelo para el cuidado, no fue tanto por la filosofía del mismo, sino que los escogieron por ser prácticos, sencillos y con un lenguaje comprensible [30]. Se habla de que el modelo de enfermería es 'ordenado y lineal', argumento contradictorio con la realidad del conocimiento, la epistemología enfermera y la forma en que ocurren los fenómenos de salud-enfermedad. Se evidencia falta de crítica sobre los fundamentos y la orientación del modelo, aunque posiblemente se debe a que en su formación profundizan en un modelo concreto, que ven como el ideal, y quizás esta visión no permite tener el espíritu crítico que da conocer varias opciones de cuidado. Aun así, por las respuestas dadas, se aprecia una actitud positiva ante los modelos enfermeros (ya recogida en investi- gaciones sobre profesionales) [3]. No obstante, esa actitud tan positiva hacia los modelos, según Benavent et al [31] obstaculiza su análisis y reflexión.

Es preciso que las escuelas universitarias dediquen un mayor esfuerzo a clarificar conceptos entre metodología y modelo enfermero: en las respuestas, los alumnos marcaban metodologías en vez de modelos enfermeros, que es lo que se les pedía. Desconocemos si confunden ambos términos (hecho bastante probable ya que no justifican por qué eligieron una metodología) o por preferencia de metodologías disociadas de marcos conceptuales. Además, parece existir una demanda de asignaturas sobre modelos y teorías de enfermería por el desconocimiento del tema que los propios estudiantes manifiestan (no los conocen en profundidad). Se deberían añadir asignaturas sobre el tema en cursos fina- 
Figura 2. Mapa conceptual del motivo de elección de un modelo teórico para la asistencia por parte de los estudiantes. Ns/Nc: no sabe/no contesta.

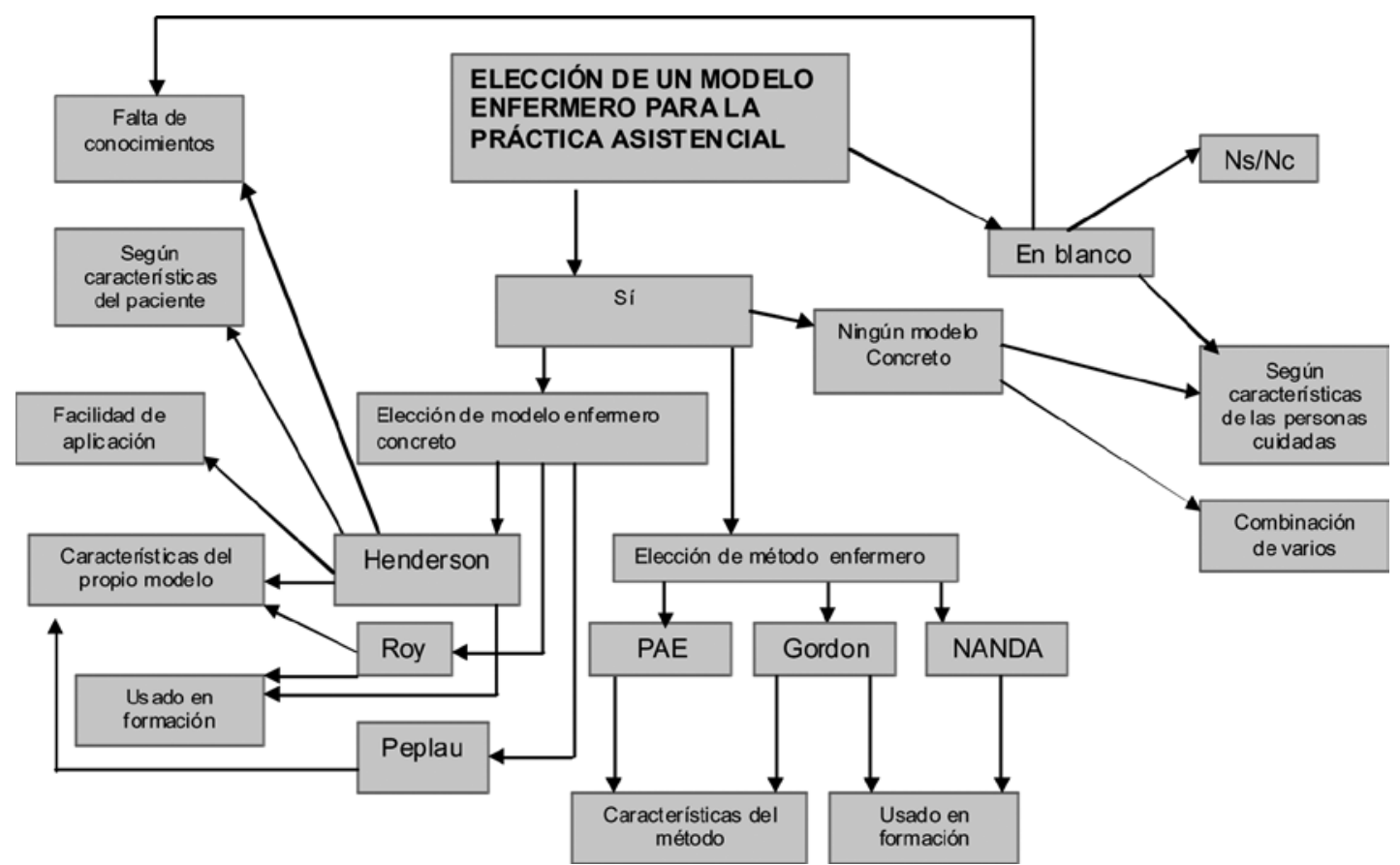

les, cuando el estudiante tiene mayor poder crítico ante posibles aplicaciones. Quizás se podría plantear el tema de las teóricas enfermeras de forma transversal, integrado en otras materias, además de propiamente en enfermería fundamental, adecuando el modelo teórico al paciente que se describe para que realmente tuviera fundamento (p. ej., se podría usar el modelo de Peplau para el abordaje de la persona en la asignatura relacionada con salud mental). En la mayoría de las escuelas españolas se usa el modelo de V. Henderson y se explican superficialmente el resto de modelos en la asignatura de 'Fundamentos de Enfermería' (primer año), por lo que falta, como ya se ha comentado, una diversificación de teorías. Posiblemente hemos de empezar por valorar la tipología de persona que frecuenta los centros hospitalarios (u otros ámbitos en los que se presta el cuidado enfermero), los recursos disponibles, su demanda (qué espera de enfermería) y qué objetivo final o resultado se pretende. Debido a este desconocimiento es lógico que el futuro enfermero elija el modelo usado en su formación, pero quizás no sea el más adecuado a las características propias de la sociedad a la que prestamos cuidado.
Resulta curioso que, dentro de los comentarios de los estudiantes, no se manifiesten quejas ya típicas en los profesionales, como son la falta de utilidad de los modelos enfermeros y la carga de trabajo, quizás porque todavía no están 'contaminados' por el mundo laboral. Sus argumentaciones son positivas hacia el uso de teoría enfermera en la práctica, ya que están en una situación inmediata a su formación [7]. Los hallazgos encontrados son similares a la primera fase del estudio de Wimpenny [4], quien entrevistó a profesionales recién titulados sobre su experiencia en modelos enfermeros. El autor concluyó que los profesionales recién titulados hablaban de la operatividad del modelo como algo que debía ser impuesto en el área clínica y que debían estar unidos al plan de cuidados y a los registros.

Aunque los profesionales (ya inmersos en el área clínica) manifestaron incertidumbre en la adaptación del modelo a la asistencia, esa opinión no se vio reflejada en los comentarios obtenidos de los estudiantes.

Las conclusiones halladas al respecto son que el modelo elegido mayoritariamente por los estudiantes es el de Virginia Henderson, que es el que guía 
su formación universitaria y resulta de fácil aplicación. Se evidencia confusión entre los términos relacionados con metodología y modelo enfermero.

\section{Bibliografía}

1. Simpson J, Taylor D. Do conceptual models of nursing work today? Can Nurse 2002; 98: 24-6.

2. López M, Santos S, Varez S, Abril D, Rocabert M, Mañé N, et al. Reflexiones acerca del uso y utilidad de los modelos y teorías de enfermería en la práctica asistencial. Enferm Clin 2006; 16: 214-7.

3. Mckenna H. The perception of psychiatric-hospital ward sisters/charge nurses towards nursing models. J Adv Nurs 1990; 15: 1319-25.

4. Wimpenny P. The meaning of models of nursing to practicing nurses. J Adv Nurs 2002; 40: 346-54.

5. Almansa P. La formación enfermera desde la sección femenina Enfermería Global 2005. URL: http://www.um.es/ojs/index. php/eglobal/article/viewFile/484/468. [15.07.2008].

6. Goulet C, Lauzon S, Ricard N. Enfermería de práctica avanzada: un tesoro oculto. Enferm Clin 2003; 13: 48-52.

7. Maben J, Latter S, Macleod J. The theory-practice gap: impact of professional-bureaucratic work conflict on newly-qualified nurses. J Adv Nurs 2006; 55: 465-77.

8. Godall M. La formación superior en enfermería del profesorado enfermero de las escuelas universitarias de enfermería. Enferm Clin 2003; 13: 26-31.

9. Consejo General de Enfermería. Libro Blanco. La aportación de la enfermería a la salud de los españoles. Situación actual y prospectiva de futuro de su desarrollo profesional. Meta 2005. Macroestudio base. Madrid: Consejo Gral. de Enfermería; 1994

10. Escuredo B. Humanismo y tecnología en los cuidados de enfermería desde la perspectiva docente. Enferm Clin 2003; 13: 164-70.

11. Medina JL. ¿Teoría? ¿Práctica? El difícil equilibrio académico y profesional en la España 'preeuropea' (II). Rev Rol Enferm 2005; $28: 246-8$.

12. McCutcheon T. Statement on nursing: a personal perspective. Gastroenterology Nursing 2004; 27: 226-9.

13. Zabalegui A. Teoría y progreso de la ciencia de enfermería. Enferm Clin 2000; 10: 263-6.

14. Fornés J. Enfermería de salud mental y psiquiátrica: planes de cuidados. Madrid: Panamericana; 2005.
15. Teixidor M. El producto enfermero y la reforma de los procesos asistenciales (I). Rev Enferm 2002; 25: 252-6.

16. López J. Propuestas para el desarrollo profesional (I). Enferm Clin 2000; 10: 114-8.

17. Alberdi R, Artigas B, Cuxart N, Agüera A. Guías para implantar la metodología enfermera. Rev Rol Enferm 2003; 26: 645-6.

18. Perfil curricular de la diplomatura de Enfermería. Terrassa: Escola Universitària Creu Roja.

19. García A. Història de l'Escola d'Infermeria 'Creu Roja' de Barcelona. Terrassa: Escola Universitària Creu Roja; 1993.

20. Memòria del curs 2005-2006 de l'Escola d'Infermeria Gimbernat. Sant Cugat: Escola Universitària d'Infermeria Gimbernat; 2006. URL: http://www.eug.es/newweb/gimbernat/ estinfo.asp?curs $=31$. [01.03.2009].

21. Aradilla A, Antonín M, Fernández P, Flor P. Competències en infermeria. Perfil formatiu basat en competències. Bellaterra: UAB; 2008.

22. Temario UIC, asignatura 'Fonaments d'infermeria I, II, III' Sant Cugat: Universitat Internacional de Catalunya. URL: http://www.uic.es/es/grado-enfermeria?fitxa_basica $=5$. [01.03.2009].

23. Zabalegui A, Cabrera E, Gallart, A, Bardallo L. Universitat Internacional de Catalunya. Àgora d'Infermeria 2004; 8: 570-1.

24. Zabalegui A. Currículum de Enfermería. Universitat Internacional de Catalunya. Sant Cugat: UIC; 1998.

25. Zabalegui A. Un programa de enfermería para la excelencia (UIC). XX Sesiones de Trabajo de la Asociación Española de Enfermería Docente. San Sebastián, marzo de 1999.

26. Rodríguez E. Atención de enfermería a un paciente con trastorno mental grave (TMG). Inquietudes 2006; 35: 30-3.

27. Román M, Campos A, Viñas C, Palop R, Zamudio A, Domingo R, et al. Las taxonomías enfermeras NANDA, NOC y NIC en la práctica asistencial hospitalaria. Enferm Clin 2005; 15: 163-6.

28. Alconero AR, Cobo JL, Casaus M, García ME, García MJ, Calvo $\mathrm{M}$, et al. Paciente intervenido de aneurisma de aorta abdominal por técnica quirúrgica endovascular. Enferm Clin 2008; 18: 96-103.

29. Marriner A, Raile M. Modelos y teorías en enfermería. 5 ed. Madrid: Mosby; 2003.

30. Atienza E, Delgado MT, Rubio MC. Aplicación práctica del modelo Roper-Logan-Tierney en la asistencia. Enferm Clin 2003; 13: 295-302.

31. Benavent MA, Francisco C, Ferrer E. Los modelos de cuidados. In Mompart MP, ed. Fundamentos de Enfermería. Colección Enfermería Siglo 21. Valencia: DAE; 2001. 